\title{
AUTOIGNITION OF HYDROGEN/AIR MIXTURES BY A THIN CATALYTIC WIRE
}

\author{
C. TREVIÑO, ${ }^{*}$ A. LIÑÁN AND V. KURDYUMOV† \\ Universidad Politécnica de Madrid \\ E.T.S.I. Aeronáuticos \\ Departamento DMT \\ Plaza Cardenal Cisneros, 3 \\ 28040 Madrid, Spain
}

\begin{abstract}
The autoignition of the catalytic reaction of hydrogen/air mixtures on thin palladium wires is analyzed in this paper. The reduced heterogeneous kinetics is modeled with a mechanism that includes the dissociative adsorption of both reactants, together with three surface reactions of the Langmuir-Hinshelwood type, and the desorption reaction of the adsorbed product, $\mathrm{H}_{2} \mathrm{O}(\mathrm{s})$. We show that for the description of the ignition conditions, this mechanism can be simplified to a single overall surface reaction involving the temperature and gas concentrations of the reactants at the surface of the wire. The resulting overall rate for the surface reaction has been tested with existing experimental results, after describing the transport of heat and reactants, by natural convection, in the gas phase for a wide range of Rayleigh numbers. The critical conditions for the catalytic ignition have been deduced using high activation energy asymptotics for the desorption kinetics of the most efficiently adsorbed reactant, $\mathrm{H}(\mathrm{s})$.
\end{abstract}

\section{Introduction}

There is an increasing interest in experimental and theoretical studies in catalytic combustion, from both practical and fundamental reasons. The catalytic combustion of hydrogen is of interest because it represents one of the simplest surface reaction schemes with a few reaction steps. It is also of importance because of the possibility of catalytic removal of $\mathrm{H}_{2}$ in nuclear plants, one of the main concerns in nuclear safety.

In general, the catalytic combustion process has been studied by either numerical simulations, using elementary chemistry [1-9], or by large activation energy asymptotic analyses, using one-step overall reaction mechanisms [10-14]. The basic principles of heterogeneous catalysis have been described elsewhere $[1,15]$. Williams et al. [1] published a model for the catalytic combustion of hydrogen at high temperatures. They presented the rate parameters for the detailed surface chemistry. Warnatz and coworkers $[2,3]$ studied, in a series of papers, the catalytic combustion and ignition of hydrogen, using detailed kinetic mechanisms for both surface and gas-phase reactions. Deutschmann et al. [4] studied the catalytic ignition of different fuels on different

*Permanent address: Facultad de Ciencias, UNAM 04510 México D. F., Mexico.

†Current address: INTA-División de Propulsión, Edificio R-02.Ctra. Ajalvir Km.4, 28850 Torrejón de Ardoz, Madrid, Spain. catalyst materials. They indicated that the ignition process is an abrupt transition from a kinetically controlled mode to one controlled by mass transport and depends mainly on the adsorption-desorption reaction steps. In their numerical simulations, they showed that one reactant or the other almost covered the surface prior to ignition. There is a series of experimental works, using a very thin catalytic wire, to obtain the critical ignition temperature of hydrogen mixtures as a function mainly of the equivalence ratio $[5,6]$. They used either a forced flow (low Reynolds number) or simply no forced flow [5]. There are other experiments involving stagnationpoint flows at high Reynolds numbers $[7,8]$. The ignition temperatures obtained using both experimental configurations differ, and a relationship between them is needed. For example, in a stagnation-point boundary layer, the heat losses depend mainly on the velocity gradient. However, when using a catalytic wire, the heat losses depend on the diameter of the wire and on the flow velocity, either forced or induced by natural convection.

In a previous work by Treviño [16], an asymptotic analysis was presented for the catalytic ignition, without any external energy supply, in a stagnationpoint flow, using a simplified model for the heterogeneous chemistry. The critical condition for ignition was deduced, giving in a closed form the parametric influence on this critical process. In this case, the ignition can be reached by increasing the temperature of the combustible gaseous mixture. When using a global one-step reaction for the surface kinetics, the reaction order for the less efficiently 
adsorbed reactant has a value of unity, while the reaction order for the most efficiently adsorbed reactant is negative, with an activation energy depending strongly in the dissociative character of the adsorption/desorption kinetics. Using the same methodology, Treviño et al. [17] studied the ignition process of a mixture of carbon monoxide and air, obtaining the critical condition for ignition, represented by a Damköhler number of the surface reaction. The analytical results were compared with the experimental data obtained with an externally heated catalytic wire and the mixture at room temperature $[18,19]$.

The objective of this work is to describe the catalytic autoignition of a mixture of hydrogen and air on a palladium catalyst, using the steady two-dimensional thin catalytic wire configuration with the flow induced by a natural convection. The critical condition for ignition is to be obtained, as represented by a critical value of the temperature of the mixture without any external energy supply to the wire. In all the experiments, the ignition condition is given by the surface temperature as a function of the fuel concentration of the mixture and, therefore, is flowgeometry dependent. We shall clarify the role of the Damköhler number of the surface reaction, which compares the rate of heat losses to the rate of heat release.

\section{Formulation}

\section{Free Convection in the Gas Phase}

A premixed combustible mixture of $\mathrm{H}_{2}$ and airwith values $T_{\infty}, Y_{\mathrm{H}_{2} \infty}$ and $Y_{\mathrm{O}_{2} \infty}$ for the ambient temperature and mass fractions of $\mathrm{H}_{2}$ and $\mathrm{O}_{2}$-is in contact with a horizontal catalytic wire of palladium with a diameter $2 a$. (Any inclination $\gamma$ from the horizontal could be included, with the gravity acceleration replaced by the normal component, $g \rightarrow g \cos \gamma$.)

The catalytic reactions within the wire lead to adsorption and desorption of $\mathrm{H}_{2}, \mathrm{O}_{2}$, and $\mathrm{H}_{2} \mathrm{O}$, corresponding, as shown below, to the overall surface reaction $2 \mathrm{H}_{2}+\mathrm{O}_{2} \rightarrow 2 \mathrm{H}_{2} \mathrm{O}$, with a rate $\omega$, to be given below in equation 18 , in moles of $\mathrm{H}_{2}$ consumed per unit surface and time; the reaction leads to a heat release $Q$ per mole of $\mathrm{H}_{2}$. The rate $\omega$ is a function of the values at the solid-gas interface of the temperature and mass fraction of the reactants.

Due to the surface chemical reaction, the temperature of the wire $T_{\mathrm{w}}$ is higher than $T_{\infty}$, producing a natural convective flow around the wire, which determines the transport of heat and mass to its surface. For the description of the flow we shall use, as in Ref. [20], the Boussinesq approximate form of the conservation equations, neglecting the effect of variations of the concentrations in the density changes, which will be written as proportional to the temperature changes times the thermal expansion coefficient $\beta=1 / T_{\infty}$. We shall use constant values for gas heat conductivity $\lambda$, thermal diffusivity $\alpha$, specific heat $c_{\mathrm{P}}$, and Prandtl, $(\mathrm{Pr})$ and Lewis numbers $\left(L e_{i}\right)$ of the species.

The temperature of the wire $T_{\mathrm{w}}$ will be considered uniform due to the typically large value of its conductivity $\lambda_{\mathrm{w}}$, when compared with the gas-phase thermal conductivity $\lambda$.

We shall consider steady conditions and that there is no external heat addition to the wire, so that the rate of heat produced by the surface reaction must be equal to the rate of the heat loss $q$ to the gas per unit length of the wire. Using cylindrical coordinates $(r, \phi)$, we have the following conditions at the wire surface

$$
\begin{aligned}
& r=a: \bar{u}=0, T=T_{\mathrm{w}} \\
& \frac{\lambda}{W_{\mathrm{H}_{2}} c_{\mathrm{P}} L_{\mathrm{H}_{2}}} \frac{\partial Y_{\mathrm{H}_{2}}}{\partial r}=\frac{2 \lambda}{W_{\mathrm{O}_{2}} c_{\mathrm{P}} L e_{\mathrm{O}_{2}}} \frac{\partial Y_{\mathrm{O}_{2}}}{\partial r}=\omega \\
& -\int_{0}^{2 \pi} \lambda \frac{\partial T}{\partial r} a d \phi=q=Q \int_{0}^{2 \pi} \omega a d \phi
\end{aligned}
$$

Here, $W_{i}$ represents the molecular weight of species $i$. The free convection problem around wires was solved in Ref. [20] for values of the Rayleigh number that are small compared with unity, which is often the case when the experiments are carried out with thin catalytic wires. Then, the size of the heated region around the wire is large compared with its radius, and the following relation

$$
\left(T_{\mathrm{w}}-T_{\infty}\right) 2 \pi \lambda / q=(1 / 3) \ln \left(g a^{3} q / \lambda T_{\infty} \alpha^{2}\right)+A_{0}
$$

is obtained for $T_{\mathrm{w}}-T_{\infty}$ in terms of $q$. Here, $A_{0}=$ 0.96 for $\mathrm{Pr}=0.72$ and $g$ is the gravity acceleration modulus. In Ref. [20], the free convection problem was also solved numerically for a wide range of values of the Rayleigh number, leading to the following correlation

$$
R a=\frac{1}{2 \pi P r N u} \exp \left(3 A_{0}-3 / N u\right) F(N u, \operatorname{Pr})
$$

between the Nusselt number $N u=q / 2 \pi \lambda\left(T_{\mathrm{w}}-T_{\infty}\right)$ and the Rayleigh number $R a=g \beta\left(T_{\mathrm{w}}-T_{\infty}\right) a^{3} /$ $\alpha^{2} \operatorname{Pr}$. The factor $F$, equal to 1 for small values of the Rayleigh number, is given by the approximate expression $F=1+18.208 N u^{2}+28.813 N u^{4}+$ 18.254 $\mathrm{Nu}^{5}$ for $\mathrm{Pr}=0.72$. The asymptotic analysis of Ref. [20] for the heat transfer to wires at small Rayleigh numbers, leading to relation 3 , can be extended to calculate, taking into account equation 1 and 2 , the difference in the mass fractions $Y_{\mathrm{i} \infty}-Y_{\mathrm{iw}}$. We thus obtain

$$
\begin{aligned}
& \left(Y_{i \infty}-Y_{\mathrm{iw}}\right) Q / c_{\mathrm{P}}=L e_{i}\left(T_{\mathrm{w}}-T_{\infty}\right) \\
& -\left(L e_{i} A_{0}-B\right) q / 2 \pi \lambda
\end{aligned}
$$

where $B=A_{0}=0.96$ for $L e=1$ and $B=0.21$ for $L e=0.3$. 
TABLE 1

Heterogeneous Reaction Model

\begin{tabular}{|c|c|c|c|c|}
\hline $\mathrm{Nr}$ & Reaction & $S$ & $A$ & $E$ \\
\hline $1 a, d$ & $\mathrm{H}_{2}+2 \mathrm{Pd}(\mathrm{s}) \rightleftharpoons 2 \mathrm{H}(\mathrm{s})$ & 0.70 & $4.8 \cdot 10^{21}$ & $84-15 \theta_{\mathrm{H}}$ \\
\hline $2 a, d$ & $\mathrm{O}_{2}+2 \mathrm{Pd}(\mathrm{s}) \rightleftharpoons 2 \mathrm{O}(\mathrm{s})$ & 0.4 & $7.1 \cdot 10^{21}$ & 230 \\
\hline $3 r$ & $\mathrm{H}(\mathrm{s})+\mathrm{O}(\mathrm{s}) \rightarrow \mathrm{OH}(\mathrm{s})+P d(\mathrm{~s})$ & - & $6.5 \cdot 10^{21}$ & 11.5 \\
\hline $4 r$ & $\mathrm{H}(\mathrm{s})+\mathrm{OH}(\mathrm{s}) \rightarrow \mathrm{H}_{2} \mathrm{O}(\mathrm{s})+P d(\mathrm{~s})$ & - & $6.5 \cdot 10^{21}$ & 17.4 \\
\hline $5 r$ & $\mathrm{OH}(\mathrm{s})+\mathrm{OH}(\mathrm{s}) \rightarrow \mathrm{H}_{2} \mathrm{O}(\mathrm{s})+\mathrm{O}(\mathrm{s})$ & - & $3.7 \cdot 10^{21}$ & 48.2 \\
\hline $6 a, d$ & $\mathrm{H}_{2} \mathrm{O}+\mathrm{Pd}(\mathrm{s}) \rightleftharpoons \mathrm{H}_{2} \mathrm{O}(\mathrm{s})$ & 0.75 & $1.3 \cdot 10^{13}$ & 44 \\
\hline
\end{tabular}

Note: Units: $A, \mathrm{~mol}, \mathrm{~cm}, \mathrm{~s} ; \mathrm{E}, \mathrm{KJ} / \mathrm{mol}$.

\section{Heterogeneous Reaction Model}

We consider a simplified model for the catalytic reactions of hydrogen and oxygen over palladium, represented by the following reaction mechanism [4] given in Table 1 . The labels $a$ and $d$ on the reactions represent adsorption and desorption, respectively. $P d(s)$ denotes a free site on the surface of the palladium catalyst. The pre-exponential factor and the activation energy apply for the desorption reactions $1 d, 2 d$, and $6 d$ and not for the adsorption reactions, which are characterized by a sticking probability. Reactions $3 r$ to $5 r$ are the surface reactions assumed to be of the Langmuir-Hinshelwood type. The adsorption kinetics are given by a sticking probability or accommodation coefficient, $S_{\mathrm{j}}$, which represents the fraction of the collisions with the surface that successfully leads to adsorption. The rate of collisions, $Z_{\mathrm{w}}$, can be computed using the classical kinetic theory as $Z_{\mathrm{w}}=p_{j} / \sqrt{2 \pi m_{j} k T}$, where $p_{i}$ and $m_{i}$ are the partial pressure and the mass of the involved species $j$, and $k$ is the Boltzmann constant, $k=1.38 \cdot 10^{23}$ $J / K$. The desorption kinetics are well represented by an Arrhenius law with a high activation energy. The concentrations of the adsorbed species are represented by their surface coverage $\theta_{\mathrm{i}}$, defined by the ratio of the number of sites occupied by the species $i$ to the total number of available sites. For the adsorbed species, we can use the steady-state approximation and, then, the governing equations are given by

$$
\begin{aligned}
\frac{d \theta_{\mathrm{H}}}{d t}= & 2 k_{\mathrm{la}} \theta_{\mathrm{V}}^{2}-2 k_{\mathrm{ld}} \theta_{\mathrm{H}}^{2}-k_{3} \theta_{\mathrm{H}} \theta_{\mathrm{O}} \\
& -k_{4} \theta_{\mathrm{H}} \theta_{\mathrm{OH}}=0 \\
\frac{d \theta_{\mathrm{O}}}{d t}= & 2 k_{2 \mathrm{a}} \theta_{\mathrm{V}}^{2}-2 k_{2 \mathrm{~d}} \theta_{\mathrm{O}}^{2}-k_{3} \theta_{\mathrm{H}} \theta_{\mathrm{O}} \\
& +k_{5} \theta_{\mathrm{OH}}^{2}=0 \\
\frac{d \theta_{\mathrm{OH}}}{d t}= & k_{3} \theta_{\mathrm{H}} \theta_{\mathrm{O}}-k_{4} \theta_{\mathrm{H}} \theta_{\mathrm{OH}}-2 k_{5} \theta_{\mathrm{OH}}^{2}=0
\end{aligned}
$$

$$
\begin{aligned}
& \frac{d \theta_{\mathrm{H}_{2} \mathrm{O}}}{d t}= k_{4} \theta_{\mathrm{H}} \theta_{\mathrm{OH}}+k_{5} \theta_{\mathrm{OH}}^{2} \\
&+k_{6 \mathrm{a}} \theta_{\mathrm{V}}-k_{6 \mathrm{~d}} \theta_{\mathrm{H}_{2} \mathrm{O}}=0 \\
& \theta_{\mathrm{H}}+\theta_{\mathrm{O}}+\theta_{\mathrm{OH}}+\theta_{\mathrm{V}}+\theta_{\mathrm{H}_{2} \mathrm{O}}=1
\end{aligned}
$$

The overall surface consumption rate of $\mathrm{H}_{2}$ in mol/ $\mathrm{cm}^{2} \mathrm{~s}$ is

$$
\omega=\Gamma k_{1 \mathrm{a}} \theta_{\mathrm{V}}^{2}-\Gamma k_{\mathrm{ld}} \theta_{\mathrm{H}}^{2}
$$

Here, $\theta_{\mathrm{V}}$ denotes the surface coverage of empty or vacant sites. All the reaction rates in equations 6 to 10 are in $1 / \mathrm{s}$ units. The reaction rates given in Table 1 can be transformed to these units to yield

$$
\begin{aligned}
k_{1 \mathrm{a}} & =\frac{S_{1} p Y_{\mathrm{H}_{2 \mathrm{w}}} W}{\Gamma W_{\mathrm{H}_{2}}^{3 / 2} \sqrt{2 \pi R T}} \\
k_{2 \mathrm{a}} & =\frac{S_{2} p Y_{\mathrm{O}_{2} \mathrm{~W}} W}{\Gamma W_{\mathrm{O}_{2}}^{3 / 2} \sqrt{2 \pi R T}} \\
k_{6 \mathrm{a}} & =\frac{S_{6} p Y_{\mathrm{H}_{2} \mathrm{O}_{\mathrm{w}}} W}{\Gamma W_{\mathrm{H}_{2} \mathrm{O}}^{3 / 2} \sqrt{2 \pi R T}} \\
k_{1 \mathrm{~d}} & =\Gamma A_{1 \mathrm{~d}} \exp \left(-E_{1 \mathrm{~d}} / R T\right) \\
k_{2 \mathrm{~d}} & =\Gamma A_{2 \mathrm{~d}} \exp \left(-E_{2 \mathrm{~d}} / R T\right) \\
k_{6 \mathrm{~d}} & =A_{6 \mathrm{~d}} \exp \left(-E_{6 \mathrm{~d}} / R T\right) \\
k_{3} & =A_{3} \Gamma \exp \left(-E_{3} / R T\right) \\
k_{4} & =A_{4} \Gamma \exp \left(-E_{4} / R T\right) \\
k_{5} & =A_{5} \Gamma \exp \left(-E_{5} / R T\right)
\end{aligned}
$$

where $\Gamma\left(\simeq 1.6603 \cdot 10^{-9} \mathrm{~mol} / \mathrm{cm}^{2}\right)$ is the surface molar concentration of active sites in $\mathrm{mol} / \mathrm{cm}^{2}$ and corresponds to the surface site density (assumed to be $10^{15}$ sites $/ \mathrm{cm}^{2}$ ) divided by the Avogadro number $\left(A v=6.02283 \cdot 10^{23}\right.$ molecules $\left./ \mathrm{mol}\right) ; R$ is the universal gas constant, and $W$ is the molecular weight of the mixture. For not very lean mixtures, $\mathrm{H}(\mathrm{s})$, almost covers the whole catalytic surface at low temperatures (below the ignition conditions). Using 
equation 8 , to eliminate reaction $5 r$, equation 7 takes the form

$$
\begin{aligned}
\frac{d \theta_{\mathrm{O}}}{d t}= & 2 k_{2 \mathrm{a}} \theta_{\mathrm{V}}^{2}-2 k_{2 \mathrm{~d}} \theta_{\mathrm{O}}^{2} \\
& -\frac{1}{2}\left(k_{3} \theta_{\mathrm{H}} \theta_{\mathrm{O}}+k_{4} \theta_{\mathrm{H}} \theta_{\mathrm{OH}}\right)=0
\end{aligned}
$$

Due to the fact that the activation energy of the desorption reaction of $\mathrm{O}(\mathrm{s}), E_{2 \mathrm{~d}}$ is extremely large, from equation (15) it is clearly seen that the reaction rate $\omega=\Gamma\left(k_{3} \theta_{\mathrm{H}} \theta_{\mathrm{O}}+k_{4} \theta_{\mathrm{H}} \theta_{\mathrm{OH}}\right) / 2$ is limited by the adsorption rate of molecular oxygen, $\omega \simeq 2 \Gamma k_{2 \mathrm{a}} \theta_{\mathrm{V}}^{2}$, where we have neglected the contribution of the desorption reaction $2 d$. Therefore, the reaction rate depends strongly on the surface coverage of empty sites, $\theta_{\mathrm{V}}$, which is determined by the interplay between adsorption and desorption of hydrogen. At low temperatures, the desorption rate of $\mathrm{H}(\mathrm{s})$ is very slow and the surface is almost covered by $\mathrm{H}(\mathrm{s}), \theta_{\mathrm{H}}$ $\rightarrow 1$. As the temperature increases, the desorption rate of $\mathrm{H}(\mathrm{s})$ also increases and so the amount of empty sites and reaction rate. The nonlinear feedback due to the exothermicity of the surface reactions produces the conditions for ignition. The system jumps abruptly from the nearly frozen or weakly reactive branch to the ignited branch, with an important change in the surface temperature.

From equations 6,11 , and 15 we obtain that

$$
\omega=\Gamma k_{1 \mathrm{a}} \theta_{\mathrm{V}}^{2}-\Gamma k_{1 \mathrm{~d}} \theta_{\mathrm{H}}^{2} \simeq 2 \Gamma k_{2 \mathrm{a}} \theta_{\mathrm{V}}^{2}
$$

or

$$
\theta_{\mathrm{V}}^{2} \simeq \frac{k_{1 \mathrm{~d}} \theta_{\mathrm{H}}^{2}}{k_{1 \mathrm{a}}-2 k_{2 \mathrm{a}}} \simeq \frac{k_{1 \mathrm{~d}}}{k_{1 \mathrm{a}}-2 k_{2 \mathrm{a}}} \text { for } \theta_{\mathrm{H}} \rightarrow 1
$$

The reaction rate $\omega$ is then given with excellent approximation by

$$
\omega=\frac{\Gamma^{2} A_{1 \mathrm{~d}} \exp \left(-E_{1 \mathrm{~d}} / R T_{\mathrm{w}}\right)}{\left[32\left(S_{1} / S_{2}\right)\left(Y_{\mathrm{H}_{2} \mathrm{w}} / Y_{\mathrm{O}_{2} \mathrm{w}}\right)-1\right]}
$$

which, aside from the factor in square brackets, representing the ratio $2 k_{2 \mathrm{a}} /\left(k_{1 \mathrm{a}}-2 k_{2 \mathrm{a}}\right)$, is only temperature dependent. The gas-phase values of the mass fraction of the reactants on the surface of the wire appear only in the first factor.

In the ignition regime, to be described below, the change in mass fractions from their ambient values are small, so that the ratio $k_{1 \mathrm{a}} / 2 k_{2 \mathrm{a}}$ can be approximated by

$$
k_{1 \mathrm{a}} / 2 k_{2 \mathrm{a}}=32\left(S_{1} / S_{2}\right)\left(Y_{\mathrm{H}_{2} \infty} / Y_{\mathrm{O}_{2} \infty}\right)
$$

or $k_{1 \mathrm{a}} / 2 k_{2 \mathrm{a}}=4\left(S_{1} / S_{2}\right) \Phi$ in terms of equivalence ratio $\Phi, \Phi=8 Y_{\mathrm{H}_{2} \infty} / Y_{\mathrm{O}_{2} \infty}$.

\section{Ignition Conditions}

Due to the large activation energy $E_{1 \mathrm{~d}}$ of the desorption reaction 1 , only a small increment in temperature above the ambient is required for ignition.
Then, we can use the approximation given above for surface reaction rate $w$ and the relation

$$
\begin{aligned}
q / 2 \pi a Q= & \omega=\left(4 \Phi S_{1} / S_{2}-1\right)^{-1} \Gamma^{2} A_{\mathrm{ld}} \\
& \exp \left(-E_{\mathrm{ld}} / R T_{\mathrm{w}}\right)
\end{aligned}
$$

can be used together with equation 4 to calculate $T_{\mathrm{w}}$. In order to obtain this, we can anticipate that in the ignition regime the temperature increment $\left(T_{\mathrm{w}}\right.$ $-T_{\infty}$ ) is of the order of the Frank-Kamenetskii temperature increment $R T_{\infty}^{2} / E_{1 \mathrm{~d}}$, required to change $\omega$ by a factor $e$ from its value at $T=T_{\infty}$. Then, the Arrhenius exponent in equation 20 can be linearized around $T_{\infty}$. If we define

$$
\varphi=\left(T_{\mathrm{w}}-T_{\infty}\right)\left(E_{1 \mathrm{~d}} / R T_{\infty}^{2}\right)
$$

the effective Rayleigh number, based on the temperature difference $\left(T_{\mathrm{w}}-T_{\infty}\right)$, can be written in the form

$$
R a / \varphi=R a^{*}=g a^{3} R T_{\infty} / E_{1 \mathrm{~d}} \alpha^{2} \operatorname{Pr}
$$

in terms of a characteristic Rayleigh number $R a^{*}$, based on the Frank-Kamenetskii temperature difference $R T_{\infty}^{2} / E_{1 \mathrm{~d}}$. Similarly, relation 20 can be written in the form

$$
\varphi N u=\Delta \exp (\varphi)
$$

in terms of the Damköhler number

$$
\begin{gathered}
\Delta=\frac{a}{\lambda} \frac{Q E_{1 \mathrm{~d}}}{R T_{\infty}^{2}}\left(4 \Phi \frac{S_{1}}{S_{2}}-1\right)^{-1} \\
\Gamma^{2} A_{1 \mathrm{~d}} \mathrm{e}^{-E_{1 \mathrm{~d}} / R T_{\infty}}
\end{gathered}
$$

When equations 22 and 23 are used together with equation 4 we obtain an explicit relation for the Rayleigh number $R a^{*}$ in terms of the Damköhler number $\Delta$ and the wire temperature $\varphi$. For fixed $R a^{*}$, two values of $\varphi$ are obtained for $\Delta<\Delta_{\mathrm{I}}$; only the lower value corresponds to a stable solution.

In order to calculate the critical value of the Damköhler number for ignition, $\Delta_{\mathrm{I}}\left(R a^{*}\right)$, we can expand $N u$ around its value at $R a^{*}$, as follows

$$
N u \simeq N u^{*}\left[1+I^{*}(\varphi-1)\right]
$$

where

$$
\begin{aligned}
& I^{*}=\left.\frac{1}{N u^{*}} \frac{d N u}{d \varphi}\right|^{*} \\
& =\frac{N u^{*}}{3-N u^{*}+\left(N u^{* 2} / F^{*}\right)(d F / d N u)^{*}} \ll 1
\end{aligned}
$$

Using this relationship for the Nusselt number, the equation determining $\varphi$ takes now the form

$$
\Delta \exp (\varphi)=N u^{*} \varphi\left[1+I^{*}(\varphi-1)\right]
$$

Equation 27 gives two values of $\varphi$ for a given $\Delta$ lower than a critical value of $\Delta_{\mathrm{I}}$ with no solution for 


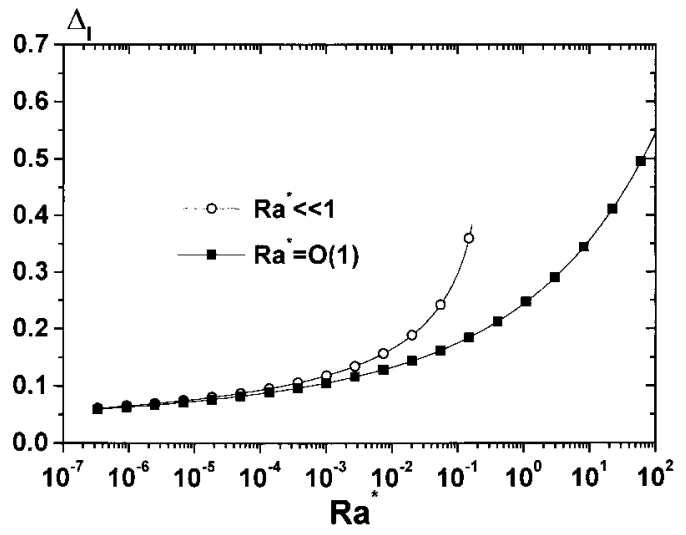

FIG. 1. The surface Damköhler number for ignition as a function of the characteristic Rayleigh number based on the Frank-Kamenetskii temperature difference, $R a^{*}$.

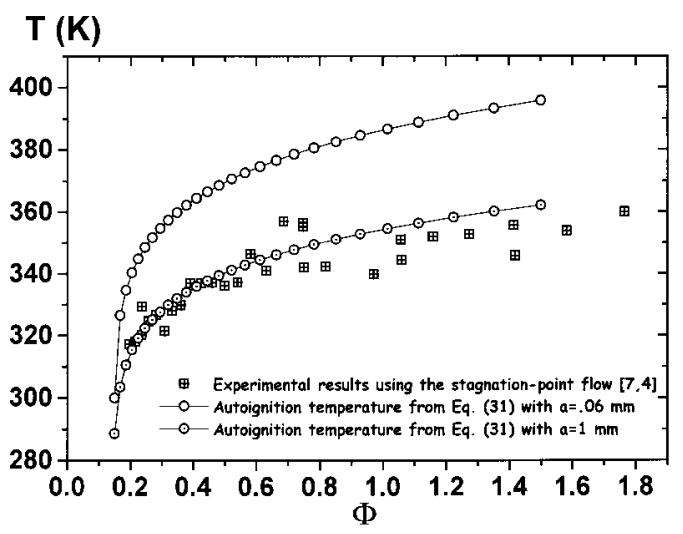

FIG. 2. The ignition temperature as a function of the equivalence ratio $\Phi$, using two different wire sizes. The experimental results reported in Ref. [4,7] obtained by using the stagnation-point flow configuration are also plotted.

$\varphi$ for values of $\Delta>\Delta_{\mathrm{I}}$. This critical value corresponds to ignition. It can be easily obtained giving

$$
\begin{aligned}
\varphi_{\mathrm{I}} & =\frac{\sqrt{1-2 I^{*}+5 I^{* 2}}-1+3 I^{*}}{2 I^{*}} \\
& \simeq 1+I^{*}+I^{* 2}+O\left(I^{* 3}\right)
\end{aligned}
$$

for $I^{*} \rightarrow 0$ and

$$
\begin{aligned}
\Delta_{\mathrm{I}} & \simeq \frac{N u^{*}\left(1+I^{*}+2 I^{* 2}\right)}{\exp \left(1+I^{*}+I^{* 2}\right)} \\
& \simeq \frac{N u^{*}}{e}\left(1+\frac{1}{2} I^{* 2}+\mathrm{O}\left(I^{* 3}\right)\right)
\end{aligned}
$$

for $I^{*} \rightarrow 0$.

\section{Results and Conclusions}

We have shown that the ignition of a reaction between $\mathrm{H}_{2}$ and air on a catalytic wire can be described in terms of an overall surface reaction $2 \mathrm{H}_{2}+\mathrm{O}_{2} \rightarrow$ $2 \mathrm{H}_{2} \mathrm{O}$ with a rate $\omega$ given by equation 18 . When the heat produced by the surface reaction is equated to the heat loss by free convection, a relation was obtained for the wire temperature in terms of the critical surface Damköhler number for ignition. The autoignition temperature of a mixture of hydrogen and air can be obtained after solving the transcendental equation

$$
\frac{\Gamma^{2} A_{1 \mathrm{~d}} \exp \left[1-E_{1 \mathrm{~d}} / R T_{\infty}\right] a Q}{\left[4 \Phi\left(S_{1} / S_{2}\right)-1\right] \lambda_{\infty} \Delta T^{*} N u^{*}\left(1+I^{* 2} / 2\right)}=1
$$

where, for the ignition regime, we have ignored the reactant consumption effects. This is justified for values of

$$
L e_{\mathrm{H}_{2}} \frac{c_{\mathrm{p}} W_{\mathrm{H}_{2}}}{Q Y_{\mathrm{H}_{2} \infty}} \frac{R T_{\infty}^{2}}{E_{1 \mathrm{~d}}} \ll 1
$$

Figure 1 shows the value of the surface Damköhler number for ignition as a function of the reference Rayleigh number, based on the Frank-Kamenetskii temperature difference, $R a^{*}$. The small Rayleigh number approximation ( $F=1$ in equation 4$)$ as well the correlation obtained for Rayleigh numbers of order unity are plotted in this figure. Important deviations occur for values of $R a^{*} \geq 10^{-4}$. Fig. 2 shows the ignition temperature as a function of the equivalence ratio $\Phi$, obtained for a wire of $0.0127 \mathrm{~cm}$ in diameter, as reported in Ref. [18]. Due to the lack of reported experimental data using a palladium wire configuration, we plotted in Fig. 2, as a reference, the experimental results reported in Ref. [4,7], giving the temperature of a palladium foil at the time of ignition, using the stagnation-point flow configuration. The qualitative comparison is good, despite the different flow configurations. The use of extremely thin wires gives higher ignition temperatures, because the heat release depends linearly on the wire radius and the heat losses depend only weakly on the size of the wire, as predicted in equation 30. On the other side, for the stagnation-point flow configuration, the heat release does not depend on the size of the foil but the heat losses depend on the employed velocity gradient. The same ignition temperature using both flow configurations can be obtained with a velocity gradient for the stagnationpoint flow proportional to $a^{-2}$. In order to have a better basis for comparison, we also plotted the resulting ignition temperature for a wire of $a=1 \mathrm{~mm}$, giving an excellent comparison with the experimental results. Fig. 3 shows the actual Rayleigh number, $R a=R a^{*} \varphi_{\mathrm{I}}$, as a function of the equivalence ratio $\Phi$ for the same experimental conditions $(a=0.0127$ $\mathrm{cm})$. The Rayleigh numbers are about 0.0004 , showing that the formula obtained for very small Rayleigh 


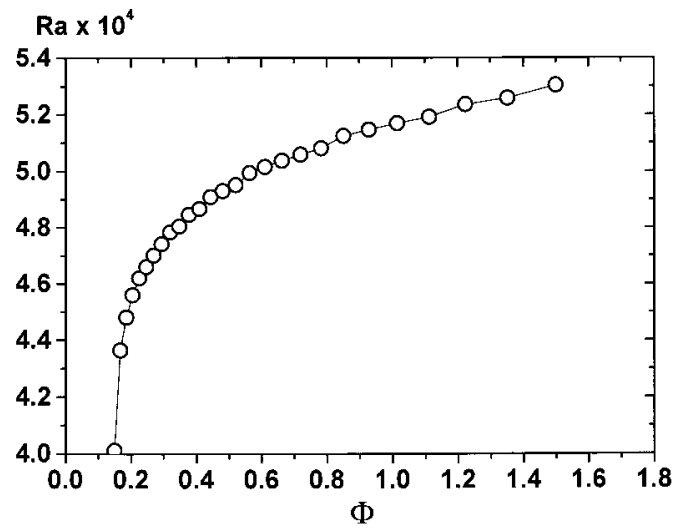

FIG. 3. The Rayleigh number at the ignition conditions as a function of the equivalence ratio $\Phi$.

numbers should not introduce important errors in the evaluation of the ignition temperature.

\section{Acknowledgments}

This work has been supported by Instituto Nacional de Técnica Aeroespacial (INTA), under Thermofluidynamics Programme number IGB4400903, and by Spanish DCICYT, under contract no. PB94-0040. C.T. acknowledges the support of CONACyT and DGAPA-UNAM of México and the Ministerio de Educación y Ciencia of Spain.

\section{REFERENCES}

1. Williams, W. R., Marks, C. M., and Schmidt, L. D., J. Phys. Chem. 96:5922 (1992).
2. Warnatz, J., Proc. Combust. Inst. 24:533 (1992).

3. Warnatz, J., Allendorf, M. D., Kee, R. J., and Coltrin, M., Combust. Flame 96:393 (1994).

4. Deutschmann, O., Schmidt, R., Behrendt, F., and Warnatz, J., Proc. Combust. Inst. 26:1747 (1996).

5. Rinnemo, M., Deutschmann, O., Behrendt, F., and Kasemo, B., Combust. Flame 111:312-326 (1997).

6. Fassihi, M., Zhdanov, V. P., Rinnemo, M., Keck, K.-E., and Kasemo, B., J. Catalysis 141:438-452 (1993).

7. Behrendt, F., Deutschmann, O., Schmidt, R., and Warnatz, J., in Heterogeneous Hydrocarbon Oxidation, ACS Symposium Series, 1996 cited in Ref. [4].

8. Fernandes, N. E., Park, Y. K., and Vlachos, D. G., Combust. Flame 118:164-178 (1999).

9. Bui, P.-A., Vlachos, D. G., and Westmoreland, P. R., Proc. Combust. Inst. 26:1763 (1996).

10. Treviño, C., and Fernández-Pello, A. C., Combust. Sci. Technol. 26:245 (1981)

11. Treviño, C., Combust. Sci. Technol. 30:213 (1983).

12. Treviño, C., and Peters, N., Combust. Flame 61:39 (1985).

13. Song, X., Williams, W. R., Schmidt, L. D., and Aris, R., Proc. Combust. Inst. 23:1129 (1990).

14. Treviño, C., and Méndez, F., Proc. Combust. Inst. 26:1797 (1996).

15. Ertl, G., Ber. Bunsen-ges. Phys. Chem. 86:425 (1982).

16. Treviño, C., Combust. Theory Model. 3:469-477 (1999).

17. Treviño, C., Prince, J. C., and Tejero, J., Combust. Flame 119:505-512 (1999).

18. Rinnemo, M., Kulginov, D., Johansson, S., Wong, K. L., Zhdanov, V. P., and Kasemo, B., Surf. Sci. 376:297 (1997).

19. Cho, P., and Law, C. K., Combust. Flame 66:159 (1986).

20. Liñan, A., and Kurdyumov, V., J. Fluid Mech. 362:199227 (1998). 